\title{
RICORDO DEL PROF. DR. HON. C. FRITZ STURM
}

\author{
m.e. GIUSEPPE GANDOLFI (*)
}

(Adunanza del 21 aprile 2016)

Il 14 marzo 2015 l'Istituto Lombardo è rimasto privo del decano dei suoi membri stranieri della Sezione di scienze giuridiche, politiche ed economiche, il Professor dottore honoris causa Fritz Sturm: giurista di ampî orizzonti, scientificamente molto fecondo, dotato di un entusiasmo, di una comunicativa e anche di uno spirito umoristico veramente non comuni.

Tedesco di nazionalità, acquisì nel 1983 la cittadinanza elvetica e considerò la Svizzera sua seconda patria. Ebbe anche intensi legami culturali e affettivi con l'Italia, la cui lingua possedeva perfettamente. Partecipò fra l'altro attivamente, dal 1990, finchè la salute glielo consentì, a tutti i convegni annuali dell'Accademia dei Giusprivatisti Europei di Pavia, della quale era un autorevole membro. E, collocato in pensione, donò all'Università di Roma-Tre tutti i libri che si trovavano nel suo studio presso la sua sede universitaria.

Nacque nel Baden, a Costanza, il 13 giugno 1929 in una famiglia di giuristi. Il padre era giudice del tribunale di prima istanza della città; la madre era consigliere superiore di prefettura. E con una giurista si unì in matrimonio. La moglie, Gudrun, conseguì il dottorato presso l'Università svizzera di Friburgo, dopo aver ottenuto la possibilità di entrare nella magistratura tedesca per due volte. Ma rinunciò, per

(*) Università degli Studi di Pavia; Istituto Lombardo Accademia di Scienze e Lettere, Milano, Italy. E-mail: accadgiuspriveur@interfree.it 
potersi dedicare allo studio scientifico del diritto; e fu autrice di varie opere, anche in collaborazione col marito.

Quando frequentava il ginnasio, Sturm non si sentiva attirato dal diritto. Aveva due diverse aspirazioni, non incompatibili: diventare pastore della chiesa riformata, e dedicarsi anche allo studio delle lingue e lettere classiche. E seguendo questa sua vocazione, durante la dittatura tedesca, che perseguitò gli ebrei e osteggiò i cristiani, egli riuniva dei suoi coetanei per leggere loro la Bibbia e commentarla.

Non si oppose tuttavia al desiderio della madre che si iscrivesse alla Facoltà di legge di Tubinga. Ed ebbe poi la possibilità di rifarsi della delusione, dovuta al mancato appagamento delle sue aspirazioni giovanili, con la sua tesi di dottorato in diritto romano, sulla 'abalienatio'. A questo concetto avevano dedicato la loro attenzione giuristi insigni; ma l'analisi delle Pandette non aveva consentito di superare l'incertezza circa l'effettiva portata del concetto stesso, rispetto alla alienatio: ossia al trasferimento volontario o involontario di beni ad altri. Si trattava di sinonimi, oppure l'abalienatio aveva per oggetto specificamente le res mancipi, ossia le cose di maggiore rilevanza socio-economica? Sturm estese la sua indagine alle testimonianze ciceroniane contenute in una delle ultime opere del grande oratore, in Topica. Concernente i "tòpoi" ossia i loci communes, i luoghi comuni quali strumenti dialiettici, questo trattato è di interesse anche giuridico, oltre che indubbiamente retorico; e fu infatti dedicato al giurista Caio Trebazio Testa. Raramente ricordata oggi, quest'opera fu oggetto di attenta considerazione nel medioevo, anche per il commento che vi dedicò Boezio, prima di perire a Pavia nelle carceri ostrogote. Con Cicerone, Sturm ebbe così a trovarsi nell'ambito da lui tanto desiderato delle lettere classiche. E si impegnò a fondo nella tesi di dottorato, che ebbe un particolare successo: furono ad essa dedicate ben diciotto recensioni, fu coronata dal prestigioso premio 'Bippert' e indusse il professor Hubert Niederländer a far sì che egli fosse subito chiamato nel 1956 all'Università di Heidelberg come assistente di diritto romano, dedito inoltre in tale veste alle lezioni di esegesi delle Pandette. In questa Università gli venne anche affidato, in quanto interprete per l'italiano e il francese, il corso di terminologia giuridica italiana nella Scuola di interpreti dell'Ateneo medesimo, corso che tenne per cinquanta semestri. A Monaco sostenne e superò nel 1964 l'esame di abi- 
litazione a cattedra con una tesi (il cosiddetto Habilitationsschrift) sulla "Stipulatio aquiliana", atto formale che consentiva di far confluire in un'unica stipulazione più obbligazioni. per poterle poi estinguere congiuntamente.

Venne, così, abilitato per le discipline di diritto romano, diritto civile, diritto internazionale privato, diritto comparato $\mathrm{E}$ nello stesso anno fu chiamato come professore straordinario di diritto romano, e successore del professor Philippe Meylan, all'Università di Losanna.

Lasciò questa sede accademica, dopo solo sette semestri, a malincuore per il largo seguito che aveva avuto fra gli studenti; anche se questo entusiasmo per il suo insegnamento aveva suscitato fra alcuni colleghi qualche gelosia, che si manifestava con l'affermazione che il diritto romano era una disciplina d'altri tempi, ormai superata. Accettò nel 1966 la chiamata all'Università di Magonza soprattutto per la prospettiva di insegnare nel suo Paese, come professore ordinario, e non solo il diritto romano, ma anche il diritto civile e il diritto internazionale privato.

Dopo un quinquennio non seppe resistere all'idea di accettare la chiamata alla cattedra offertagli dall'Università di Marburgo, celebre anche perchè in essa aveva insegnato Friedrich Karl von Savigny. Erano però gli anni Settanta e l'atmosfera aveva iniziato, con la contestazione studentesca, a guastarsi. Egli venne così a trovarsi esposto alla prepotenza come un parafulmine, in quanto decano della Facoltà di giurisprudenza, considerata dagli agitatori reazionaria più delle altre facoltà, soprattutto per l'insegnamento del diritto romano e del diritto civile, dunque del codice civile tedesco, il famoso $B G B$. Promulgato alla fine dell'Ottocento, questo glorioso codice, in quanto di impronta romanistica e moderatamente liberale, già era stato osteggiato dal regime nazista che intendeva sostituirlo con un Volksgesetzbuch, un codice del popolo, conforme a una pretesa tradizione giuridica autenticamente germanica. In effetti, come scrive Franz Wieacker, questo regime ebbe solo a disarticolarne e sostituire parzialmente dei settori normativi in sè coerenti. $\mathrm{Ma}$ - tornando ai disordini che dovette affrontare a Marburgo - Sturm in un suo scritto autobiografico parla di infiltrati, fra i contestatori, che erano pilotati dalla organizzazione Spartacus, teleguidata dalle autorità della Germania dell'Est. Qui era allora in fase di redazione il Zivilgesetzbuch, il codice civile che sarebbe ivi subentrato al $B G B$ nel 1976: per "l'evoluzione dei cittadini in personalità socialiste" come 
in esso recita il paragrafo 1 . In questa situazione, Sturm dovette organizzare un gruppo di studenti neutrali, coraggiosi, per cercare di proteggere dei colleghi impediti con la violenza di tenere le loro lezioni; e ciò perchè il rettore, socialista, si rifiutava di far intervenire la polizia. Cosicchè nelle strade di Marburgo apparvero dei manifesti con la scritta: "Im Kopf des Sturm ist der Wurm!" (nella testa di Sturm c'è un 'verme' o un 'drago', se mai volessimo intendere questa parola nel significato che ha nell'antica mitologia germanica). Se non accolse l'offerta della cattedra di diritto romano che gli fece l'Università di Berna, fu per non abbandonare la nave, come egli ha scritto, nel momento in cui correva il rischio di affondare. Ed ebbe a chiedersi: "insomma l'origine del mio cognome (Sturm) non evoca forse l'idea della presenza in prima linea"? Ma la situazione si aggravò. Le autorità governative, nel tentativo di arginare la pesante protesta degli studenti, ritennero necessario adottare dei provvedimenti di impronta populistica, come la cogestione e la partecipazione agli organi accademici di studenti e impiegati, misure che in realtà ebbero a minacciare - sono parole di Sturm - "la libertà dell'insegnamento e della ricerca".

Egli pertanto, nel 1977, accettò la chiamata dell'Università di Losanna per l'insegnamento del diritto tedesco, che era stato ivi tenuto per parecchi anni dal professor Otto Riese. Tornò con gioia nella Suisse romande dove, sulla sponda nord del lago di Ginevra, il Lemàno, e con vista quanto mai suggestiva sul lago e sulle Alpi, aveva ancora quella bellissima villa che aveva comprato negli anni Sessanta; e dove formò una ricchissima biblioteca e ospitò colleghi e studenti. Restò a Losanna definitivamente; e dunque dei suoi quarant'anni di insegnamento ne trascorse qui ventitre. E ben a ragione considerava la Svizzera, come dicevo, la sua seconda patria. Dove ebbe la grande soddisfazione che numerosi studenti venissero dalla Germania per apprendere da lui il diritto tedesco! Il che, come egli confessava, non mancò di suscitare anche qui qualche gelosia in alcuni suoi colleghi. Ma il suo stile, il suo entusiasmo, la sua disponibilità valsero a conquistargli anche una particolare considerazione presso gli studenti svizzeri e francesi. Si intratteneva amabilmente e a lungo con gli allievi dopo le lezioni, ed organizzava per loro dei viaggi di cultura: a Berlino, Roma, KarlsruheHeidelberg. Molto simpatiche ma anche istruttive per gli studenti erano le cene collettive con lui al termine dei semestri accademici. Sturm 
ricorda di aver ricevuto la telefonata della madre di un suo ex allievo, che desiderava informarlo di un buon risultato professionale del figlio e che gli disse anche: "Professore, mio figlio temeva i suoi professori per il loro atteggiamento spesso glaciale; ed è stato grazie al contatto con Lei che il ghiaccio si è rotto!".

Ma il successo che riscuoteva era dovuto anche, e soprattutto, al metodo e all'efficacia del suo insegnamento. Piuttosto che tenere lezioni ex cathedra egli, avvalendosi delle risorse offerte dalla maieutica, stimolava delle riflessioni e discussioni volte ad individuare le soluzioni di casi pratici, che egli desumeva dall'esperienza giurisprudenziale o da casi della vita quotidiana; e formava così gli allievi sul piano pratico, oltre che dottrinale, preparandoli idoneamente per la magistratura e la professione.

Ma la sua personalità emergeva, oltre che nel campo didattico, come abbiamo visto, anche e soprattutto nel campo della ricerca scientifica, alla quale si è encomiabilmente dedicato, ben può dirsi, sino agli ultimi momenti della sua vita. Vanno in primo luogo sottolineate la rigorosa impostazione che adottava nelle sue indagini e la limpidezza dello stile con cui ne esprimeva i risultati. Egli inoltre rifuggiva da quelle inutili divagazioni delle quali amano compiacersi quegli autori che intendono accattivarsi tout court la simpatia di tutti i lettori, anche quelli indifferenti, ma con un linguaggio sostanziale puntava decisamente al nocciolo di ogni problema, per sviscerarlo e risolverlo in modo conforme ai dati del diritto e del fatto.

La sua produzione scientifica, già l'ho accennato, è vastissima. Fra monografie, corsi didattici, saggi, commentari, note a sentenze, recensioni, traduzioni di leggi straniere e di opere di giuristi, commemorazioni, note su attualità e varietà giuridiche: sono più di seicentocinquanta i suoi scritti, e l'ultimo è del 2015, l'anno della sua scomparsa. Vengono ora ammirevolmente raccolti dalla sua carissima Gudrun, pure coautrice di taluni di essi, come già dicevo, e che sta pensando a riedizioni per tematiche omogenee.

Accennavo alla vastità degli orizzonti culturali di Sturm: quale emerge dal fatto che i suoi lavori attengono al diritto romano, alla storia del diritto, al diritto civile (tedesco, francese, svizzero, italiano, austriaco, angloamericano, europeo), al diritto internazionale privato, al diritto comparato, all'unificazione del diritto in Europa. Fra le sue opere 
civilistiche, molte riguardano temi di grande attualità, come il diritto delle persone lato sensu inteso, e anzitutto la facoltà di scegliere il nome, e la libertà stessa dunque di sceglierlo; temi concernenti il cognome della moglie, la nazionalità, la cittadinanza, l'obbligo del mantenimento e degli alimenti, la tutela dei minori e degli incapaci, l'adozione, gli atti dello stato civile, fra l'altro.

Oltre alla sua lingua materna, parlava perfettamente il francese, l'italiano, l'inglese e riusciva ad esprimersi in olandese e in spagnolo. Il che gli consentì di tenere anche corsi universitari, seminari, conferenze in molte Università, con grande successo. Voglio ricordare che alla Statale di Milano svolse un corso di diritto comparato come professore a contratto, e suscitò tale interesse e simpatia che alla fine gli studenti omaggiarono lui con una bottiglia di vino molto pregiato e sua moglie Gudrun con un grande mazzo di fiori. Ma questa sua presenza in diversi continenti non cessò dopo la sua nomina a professore onorario, avvenuta il $1^{\circ}$ settembre 1999 . Tenne successivamente relazioni scientifiche ai congressi di Graz, Catania, Clermont-Ferrand, Karlsruhe, Bochum, Treviri; svolse dei corsi come Gastprofessor a Catanzaro, Ferrara, Urbino, Tokyo, Trento; fece numerose conferenze nei convegni di aggiornamento per ufficiali di stato civile a Magdeburgo, Saarbrücken, Bayreuth, Amburgo, Wörlitz, Nordwijkerhout, Merano: ma è questa un'elencazione incompleta.

Fu membro del nostro Istituto Lombardo e della già menzionata Accademia dei Giusprivatisti Europei. Ebbe pubblici riconoscimenti, non solo accademici; accenno al titolo di dottore bonoris causa conferitogli dall'Università di Liegi, alla decorazione del merito di prima classe della Repubblica Federale Tedesca, alla raccolta internazionale di scritti in suo onore, che gli venne presentata in una solenne, festosa cerimonia.

La sua scomparsa è dunque una grave e dolorosa perdita: per la scienza giuridica e per una vastissima cerchia di amici, colleghi, discepoli, estimatori, ai quali egli molto ha dato, con il suo lavoro scientifico, ma anche con il suo calore umano, con il suo fervido entusiasmo, con la simpatia che esprimeva e sapeva suscitare. Ed è in questa luce che ora mi è caro ricordarlo, nella sua eccezionale vitalità! 